\title{
Conocimiento y Evaluación Acerca de las Mutuales de Seguridad en Chile
}

\author{
PUBLIC KNOWLEDGE AND EVALUATION OF OCCUPATIONAL SAFETY AND HEALTH INSURANCE INSTITUTIONS \\ IN CHILE
}

\author{
Juan Carlos Oyanedel ${ }^{1}$, Héctor Sánchez ${ }^{2}$, Manuel Inostroza ${ }^{2}$, Camila Mella ${ }^{3}$, Salvador Vargas ${ }^{3}$ \\ 1. Departamento de Gestión y Políticas Públicas, Facultad de Administración y Economía, Universidad de Santiago de Chile. \\ 2. Instituto de Salud Pública, Universidad Andrés Bello. Santiago, Chile. \\ 3. Facultad de Administración y Economía, Universidad de Santiago de Chile.
}

\begin{abstract}
RESUMEN
Este artículo presenta el nivel de conocimiento y evaluación de las mutuales de seguridad, aseguradoras privadas de accidentes laborales y enfermedades profesionales en Chile. Se aplicó una encuesta telefónica a 300 trabajadores dependientes residentes en hogares ubicados en las zonas urbanas de Santiago de Chile. Los hogares fueron seleccionados de forma aleatoria sobre la base de un listado público de teléfonos de hogares. Los resultados muestran que la mayor parte de los encuestados sabe en qué consisten estas instituciones, pero aproximadamente un tercio afirma no tener conocimiento del sistema. Las evaluaciones más altas respecto a la información recibida de las mutuales están en el área de beneficios no relacionados al trabajo (por ejemplo, descuentos en establecimientos educacionales y tiendas) y a las coberturas en salud en caso de accidente. Para aquellos usuarios que han sufrido un accidente laboral, las valoraciones más altas están en el área de atención médica y rehabilitación, mientras las más bajas se encuentran en las prestaciones asociadas a traslado hacia los centros asistenciales, así como de los procedimientos administrativos asociados al tratamiento del accidente. Se concluye que resulta necesario promover el conocimiento de las actividades de las mutuales y mejorar las estrategias de promoción de actividades de prevención de accidentes ocupacionales con el objeto de hacer más eficiente su rol público. De igual manera, la información recolectada permite identificar los procedimientos de apoyo al tratamiento de accidentes como una de las áreas de mejora en el marco de la atención de salud ocupacional.
\end{abstract}

(Oyanedel J, Sánchez H, Inostroza M, Mella C, Vargas S, 2014. Conocimiento y Evaluación Acerca de las Mutuales de Seguridad en Chile. Cienc Trab. Sep-Dic; 16 [51]: 146-151).

Palabras claves: SALUD LABORAL, ACCIDENTES LABORALES, MUTUALES DE SEGURIDAD, OPINIÓN PÚBLICA, ESTUDIO TRANSVERSAL.

\section{ABSTRACT}

This article presents the level of public knowledge and evaluation towards Mutuales de seguridad -private occupational safety and health insurance institutions- in Chile. The sample is composed by phone interviews to 300 adult dependent workers living in households located in urban areas of Santiago de Chile. Households were selected through random dialling. The results show a high proportion of workers declaring to know what these institutions are, while about a third declares not to have information about them. Regarding information provided by mutuales, they report higher satisfactionwith information associated to benefits non-related to health and safety (for instance, discounts) and with those associated with the coverage in case of accident. For those who have suffered an occupational accident, the most valued areas of the service are the medical attention and the rehabilitation process, while the least valued areas are the process of transportation from the site of accident to the medical centre and the administrative process associated to the accident's treatment. These results highlight the need for better strategies to promote activities aiming to prevent occupational accidents. They also make clear that there is space for improvement in the area of support activities related to accidents treatment.

Key words: OCCUPATIONAL HEALTH, OCCUPATIONAL ACCIDENTS, OCCUPATIONAL SAFETY AND HEALTH INSURANCE, PUBLIC OPINION, CROSS-SECTIONAL STUDY.
Correspondencia / Correspondence:

Juan Carlos Oyanedel, PhD

Facultad de Administración y Economía, Universidad de Santiago de Chile Fanor Velasco 43. Santiago, Chile

Tel.: +56 (9) 94327972.

e-mail: juan.oyanedel@usach.cl

Recibido: 12 de Junio 2014 / Aceptado: 22 de Octubre 2014

\section{INTRODUCCIÓN}

El sistema de salud en Chile se caracteriza por la presencia de actores privados y públicos en la provisión de prestaciones sanitarias. Las reformas llevadas a cabo en la década de 1980, consagraron a dos instituciones como las más relevantes en esta materia $^{1,2}$. Una de ellas es el Fondo Nacional de Salud (FONASA), a cargo del Estado a través del Ministerio de Salud, mientras que la segunda está conformada por las Instituciones de Salud Previsional (ISAPRE). Los cotizantes deben abonar un porcentaje mínimo de su salario, que en el caso del sistema público es fijo 
(7\%), mientras que en el sector privado fluctúa de acuerdo al contrato específico que se suscriba, el cual depende del riesgo y los beneficios que involucre. Aquellas personas sin capacidad monetaria de cotizar son incluidas en FONASA automáticamente, pudiendo optar a beneficios en salud.

Las ISAPRE pueden ser abiertas o cerradas ${ }^{3}$, donde las primeras son aquellas disponibles para cualquier cotizante que pueda pagar alguno de los planes que estas instituciones ofrecen. Las segundas, por otra parte, se caracterizan por estar limitadas a un determinado grupo de trabajadores, ya sea por su pertenencia a alguna empresa o rama de la producción. Un ejemplo de este tipo de aseguradora lo constituyen las asociadas a las divisiones administrativas de la Corporación Nacional del Cobre (CODELCO).

Un tercer actor involucrado está conformado por las mutuales de seguridad ${ }^{4,5,6}$, que se ocupan sólo de cubrir prestaciones asociadas a accidentes laborales y enfermedades profesionales. Estas nacen en la década de 1950, consolidándose legalmente en 1968. Las mutuales se caracterizan por ser instituciones privadas sin fines de lucro, existiendo tres en Chile: Asociación Chilena de Seguridad, Mutual de Seguridad e Instituto de Seguridad del Trabajo.

Este tipo de aseguradora se torna particularmente importante dados los antecedentes que existen tanto a nivel global como nacional referido a accidentabilidad laboral. De acuerdo a estimaciones realizadas para América Latina, la tasa de fatalidad producto de causas relacionadas con el trabajo es 17,2 por cada 100.000 trabajadores, mientras que para Chile dicha tasa rondaría los 14,7. En el caso de los accidentes no fatales, estos alcanzan una tasa de 13.192 por cada 100.000 trabajadores para América Latina mientras que la tasa de Chile es de 11.182 por cada 100.000 habitantes $^{7}$. Desde una perspectiva longitudinal, los accidentes y enfermedades con resultado de muerte han aumentado en términos absolutos, pero las tasas han disminuido ${ }^{8}$.

A nivel latinoamericano, existe una alta incidencia de accidentes relacionados con el trabajo, aun cuando el reporte sería menor a la cifra real. Las principales razones que se aducen para explicar estos niveles de accidentabilidad se relacionan con el riesgo asociado a determinadas actividades económicas, así como a la falta de esfuerzos preventivos por parte de empleadores y gobiernos ${ }^{9}$.

En Chile, entre el año 2002 y el 2012, la tasa de accidentabilidad disminuyó aproximadamente dos puntos porcentuales, desde un $8,72 \%$ a un $5,99 \%$. A su vez, se observa que este decrecimiento no manifiesta retrocesos en el período señalado ${ }^{10-11}$. Los datos de la Primera Encuesta Nacional de Empleo, Trabajo, Salud y Calidad de Vida de los Trabajadores y Trabajadoras en Chile ${ }^{12}$ señalan que un $6,4 \%$ de los hombres y un 6,2\% de las mujeres sufrieron un accidente laboral durante los últimos doce meses, mientras que al segmentar por zona geográfica de residencia las personas del área urbana tuvieron una incidencia de un 6,6\%, mientras que las de áreas rurales, un 4,0\%. De acuerdo a estos datos, los trabajadores de niveles socioeconómicos más bajos son los más afectados por este tipo de dolencias.

A su vez, los antecedentes presentados por esta misma encuesta dan cuenta de que las enfermedades que fueron reconocidas como profesionales alcanzan al 29,5\% del total. Un dato relevante es que, en este tipo de dolencia, el reconocimiento es ostensiblemente mayor en los patrones o empleadores $(93,2 \%)$ que en el resto de las categorias ocupacionales, siendo la de menor reconocimiento los trabajadores por cuenta propia.

La importancia de la temática de la prevención y tratamiento de accidentes laborales y enfermedades profesionales, de acuerdo a los datos objetivos presentados, resulta manifiesta. Sin embargo, una pregunta que necesariamente debe plantearse, y que se erige como el objetivo de la presente investigación, es si los propios trabajadores conocen las instituciones que tienen a su cargo estas funciones, así como la evaluación que de estas hacen.

Este problema adquiere particular relevancia en la medida de que los empleadores tienen fuertes incentivos económicos para subdeclarar los accidentes laborales, en tanto que las empresas deben financiar el sistema de mutuales de seguridad de acuerdo a la accidentabilidad de sus trabajadores ${ }^{13}$. A su vez, las transformaciones de la estructura social chilena, con una fuerte expansión del sector terciario ${ }^{14}$, conllevan un aumento de riesgos psicosociales, asociadas a la utilización flexible del tiempo, riesgos ergonómicos y salud mental ${ }^{15}$.

Asimismo, el punto nodal que justifica la contribución del estudio que se presenta dice relación con la falta de especificidad acerca de este tipo de aseguradoras en las diferentes investigaciones realizadas a través de encuestas, en la medida que se concentran en aspectos asociados a percepciones acerca de la seguridad y salud del lugar de trabajo ${ }^{16}$, así como en las inconsistencias entre la preocupación sobre el tema y las escasas acciones coherentes con ella ${ }^{17}$. En materia de políticas públicas el estudio sobre esta área puede incidir en una mayor cobertura ante accidentes laborales y enfermedades profesionales, fruto del conocimiento que se puede generar una vez que se diagnostique adecuadamente el estado actual entre los trabajadores chilenos.

De este modo, el artículo presenta a continuación la metodología utilizada para abordar el problema de investigación. Posteriormente, se presentan los principales resultados, enfatizando el conocimiento y evaluación de las mutuales de seguridad. Finalmente, se concluye destacando la necesidad de seguir avanzando en materia informativa para efectos de optimizar la seguridad laboral en Chile.

\section{MATERIALES Y MÉTODOS}

La población del estudio corresponde a trabajadores dependientes mayores de 18 años residentes en el Gran Santiago. Se escogió este grupo en la medida que es el directo beneficiario de las aseguradoras de salud como las mutuales. La muestra fue seleccionada aleatoriamente del listado público de teléfonos de hogares particulares de la ciudad de Santiago. El procedimiento incluyó, en primer lugar, el contacto con el hogar; y, posteriormente, la aplicación de la entrevista a un trabajador dependiente del hogar seleccionado de forma aleatoria. La muestra alcanzó un total de 300 casos, los que fueron ponderados por sexo, nivel socioeconómico y edad, con el objeto de obtener así un error de $\pm 5,6 \%$, para un nivel de confianza del 95\%.

Tabla 1.

Distribución de la muestra.

$\begin{array}{lcc}\text { Sexo } & \text { Hombre } & 43 \% \\ & \text { Mujer } & 57 \% \\ & \text { ABC1 } & 11 \% \\ \text { Nivel Socioeconómico } & \text { C2 } & 22 \% \\ & \text { C3 } & 28 \% \\ & D & 39 \% \\ \text { Edad } & 18-30 & 31 \% \\ & 31-45 & 25 \% \\ & 46-59 & 24 \% \\ & 60 \text { o más } & 20 \%\end{array}$


La recolección de datos se realizó a través de un cuestionario estructurado con preguntas cerradas, elaborado luego de una revisión de antecedentes empíricos y teóricos, además de consulta a expertos en materia de seguridad laboral y sistema de salud. La aplicación de la batería de preguntas se efectuó vía telefónica, durante el mes de marzo de 2013, previa capacitación de un equipo de encuestadores para la correcta consecución de esta fase de la investigación. Los datos fueron analizados a través del paquete estadístico IBM-SPSS v. 20.

Las variables que se seleccionaron para el análisis corresponden a nueve dimensiones: conocimiento de mutuales; afiliación; beneficios; utilización de servicios; fuentes de información; evaluación de la información proporcionada sobre las mutuales; accidentabilidad; complejidad de prestaciones; y, evaluación del servicio. Las razones que llevaron a escoger los indicadores de estos factores radican en que el desconocimiento es una barrera importante para no acceder a atenciones de salud en caso de accidentes laborales o enfermedades profesionales, tal como evidencia la Encuesta Nacional de Empleo, Trabajo, Salud y Calidad de Vida de los Trabajadores y Trabajadoras en Chile $^{12}$. Así, la percepción y, sobre todo, el conocimiento acerca de las mutuales es un elemento de suma importancia para mejorar la salud de los trabajadores.

El análisis que se presenta da cuenta de una aproximación descriptiva y exploratoria, constituyendo una primera revisión empírica a este tema.

\section{RESULTADOS}

En primer lugar, es posible constatar que, del total de encuestados, el 66\% manifiesta conocer en qué consisten las mutuales de seguridad. Del total, el 51\% de ellos declara que la empresa en donde actualmente trabaja "no" se encuentra afiliada a alguna de las mutuales existentes. Por otra parte, del 49\% que declara que su empresa "sí" está afiliada, el 28\% lo está en la Asociación Chilena de Seguridad (ACHS); el 12\% al Instituto de Seguridad del Trabajo (IST); y, finalmente, el 9\% en la Mutual de Seguridad. A su vez, el $63 \%$ declara que "no" conoce cuáles son los beneficios y cobertura de las mutuales de seguridad.

En otra dimensión, el 69\% declara "no" haber utilizado alguno de los servicios proporcionados por su mutual. Por otra parte, dentro del 33\% que "sí" los conoce, el 23\% lo hace por la "atención por enfermedades profesionales"; un 4\% señala que "ha utilizado otras prestaciones"; un 3\% que ha recibido "atención por enfermedades comunes" e igual porcentaje ha recibido "capacitación en prevención de riesgos".

Del total de personas que se han atendido en alguna mutual de seguridad, el 38\% supo de ese derecho a través de "capacitaciones realizadas por la misma mutual", le sigue un 19\% que declara haberse enterado a través de "otros medios"; un 17\%, que sostiene que supo gracias a "compañeros de trabajo"; un 16\% que señala que se enteró mediante "representantes de la mutual en la empresa"; un 7\% a través del "prevencionista de riesgos de su empresa”; y, finalmente, sólo el 3\% declara que se enteró a través de "medios de comunicación". (Tabla $\mathrm{N}^{\circ} 2$ ).

Asimismo, los encuestados que han recibido atención en alguna mutual de seguridad evalúan con una nota promedio de 5,4 la información entregada sobre los programas de beneficios (en escala de 1 a 7 , donde 1 es "muy malo" y 7 "muy bueno"). Le sigue, la "cobertura de salud por enfermedad laboral", con un
Tabla 2.

Resultados.

\begin{tabular}{|c|c|c|}
\hline $\begin{array}{l}\text { ¿Sabe en qué consisten las } \\
\text { mutuales de seguridad }\end{array}$ & $\begin{array}{l}\text { No } \\
\text { Si }\end{array}$ & $\begin{array}{l}34 \% \\
66 \%\end{array}$ \\
\hline $\begin{array}{l}\text { ¿Sabe si su empresa está afiliada } \\
\text { a alguna mutual de seguridad? }\end{array}$ & $\begin{array}{l}\text { No } \\
\text { Si, Instituto de Seguridad } \\
\quad \text { del Trabajo (IST) } \\
\text { Si, Asociación Chilena de Seguridad } \\
\text { (ACHS) } \\
\text { Si, Mutual de Seguridad } \\
\text { (Cámara Ch. de la Construcción) } \\
\text { Si, Instituto de Seguridad Laboral (ISL) }\end{array}$ & $\begin{array}{l}9 \% \\
0 \%\end{array}$ \\
\hline $\begin{array}{l}\text { ¿Sabe cuáles son los beneficios y } \\
\text { cobertura de las mutuales } \\
\text { de seguridad? }\end{array}$ & $\begin{array}{l}\text { No } \\
\text { Si }\end{array}$ & $\begin{array}{l}63 \% \\
37 \%\end{array}$ \\
\hline $\begin{array}{l}\text { ¿Alguna vez ha utilizado alguno } \\
\text { de los servicios promocionados } \\
\text { por su mutual? }\end{array}$ & $\begin{array}{l}\text { No } \\
\text { Si }\end{array}$ & $\begin{array}{l}69 \% \\
31 \%\end{array}$ \\
\hline $\begin{array}{l}\text { ¿Cómo supo que tenía derecho } \\
\text { a ser atendido por la mutual? }\end{array}$ & $\begin{array}{l}\text { Capacitaciones realizadas por la mutual } \\
\text { Representante de la mutual en la empresa } \\
\text { Prevencionista de riesgos } \\
\text { Compañeros/as de trabajo } \\
\text { Medios de Comunicación } \\
\text { Otro }\end{array}$ & $\begin{array}{c}38 \% \\
16 \% \\
7 \% \\
17 \% \\
3 \% \\
18 \%\end{array}$ \\
\hline $\begin{array}{l}\text { ¿Qué nota le pone a la información } \\
\text { que la mutual le entrega en...? }\end{array}$ & $\begin{array}{l}\text { Programa de beneficios } \\
\text { Cobertura de salud por enfermedad labora } \\
\text { Cobertura de salud por enfermedad comúr } \\
\text { Beneficios familiares } \\
\text { Capacitación en prevención de riesgos }\end{array}$ & $\begin{array}{r}5,4 \\
\text { al } 5,3 \\
4,4 \\
3,2 \\
4,1\end{array}$ \\
\hline $\begin{array}{l}\text { Durante los últimos } 12 \text { meses, } \\
\text { ¿ha sufrido alguna enfermedad } \\
\text { o accidente laboral? }\end{array}$ & $\begin{array}{l}\text { No } \\
\text { Sí }\end{array}$ & $\begin{array}{l}81 \% \\
19 \%\end{array}$ \\
\hline $\begin{array}{l}\text { Con respecto a la prestación de } \\
\text { servicios entregada, } \\
\text { ¿cómo describiría el proceso? }\end{array}$ & $\begin{array}{l}\text { Complicado, ningún compañero/a de } \\
\text { trabajo sabia qué hacer } \\
\text { Complicado, hubo que comprobar ante } \\
\text { la mutual que fue un accidente laboral } \\
\text { Sencillo, existe un protocolo claro en la } \\
\text { empresa para el traslado de } \\
\text { accidentados a la mutual } \\
\text { Sencillo, existe un protocolo claro en la } \\
\text { empresa para el traslado de accidentados } \\
\text { a la clinica/consultorio } \\
\text { Otro }\end{array}$ & $\begin{array}{c}2 \% \\
16 \%\end{array}$ \\
\hline $\begin{array}{l}\text { ¿Qué nota le pone a los siguientes } \\
\text { elementos de la atención? }\end{array}$ & $\begin{array}{l}\text { Tiempo de espera para traslado } \\
\text { Calidad del traslado } \\
\text { Procedimientos administrativos } \\
\text { Recepción en el centro de salud (trato) } \\
\text { Calidad de la atención médica recibida } \\
\text { Programa de rehabilitación } \\
\text { Según experiencia }\end{array}$ & $\begin{array}{l}4,8 \\
3,4 \\
4,8 \\
5,3 \\
5,8 \\
6,2 \\
5,5\end{array}$ \\
\hline
\end{tabular}

promedio de 5,3; la "capacitación de prevención de riesgos" con un promedio de 4,1; y la "cobertura de salud por enfermedad común" con un promedio de 4,0. Al respecto, cabe precisar que la calidad de la información entregada respecto a los "beneficios familiares" obtiene nota deficiente, con un promedio de 3,0.

Por otra parte, el 19\% de los entrevistados señala haber sufrido alguna enfermedad o accidente laboral durante el último año. En lo referente a la prestación entregada a raíz de estas situaciones, del total de encuestados que padecieron alguno, el 57\% dice que su atención fue "sencilla, debido a la existencia de un protocolo claro para el traslado de accidentados a la mutual”. Le sigue el 18\% que señala que su atención fue "complicada, debido a que hubo que comprobar ante la mutual que fue un accidente laboral"; el $12 \%$ que sostiene que "otro" fue el procedimiento de atención; el $11 \%$ que señala que la atención fue "complicada, ya que ningún compañero de trabajo sabía qué hacer”; y, finalmente, sólo el $2 \%$ 
manifestó que fue "sencilla, pues existe un protocolo claro para el traslado de accidentados a la clínica/consultorio".

A su vez, los encuestados que han recibido atención por algún accidente o enfermedad laboral evalúan con nota promedio de 5,0 el "tiempo de espera para traslado" y la "recepción en el centro de salud; y con un 4,8 los "procedimientos administrativos de atención". Cabe señalar, que la "calidad del traslado" obtiene nota deficiente, con un promedio de 3,0.

Finalmente, las personas que han recibido atención por algún accidente o enfermedad laboral evalúan con nota 6,0 (como promedio) la "calidad de la atención médica recibida" y el "programa de rehabilitación”. En términos generales, la "experiencia de atención" recibe nota 5,0 como promedio.

\section{DISCUSIÓN}

Los resultados explicitados presentan un panorama complejo sobre el rol de las mutuales de seguridad en Chile. Los resultados muestran una positiva evaluación respecto a la información que estas prestan, la sencillez en la atención y su calidad general. Señalan también que la propia acción de las mutuales es la reportada como más importante para conocer los beneficios que proveen estas instituciones, en tanto que las capacitaciones que realizan son el medio más frecuentemente señalado en dicho ítem.

No obstante, la otra cara de la moneda indica que un tercio de los trabajadores no conoce la existencia de las mutuales y, más aún, el 51\% de ellos señala no conocer si su empresa está o no asociada a una. Esto constituye, sin duda, una limitación al ejercicio de los derechos de los trabajadores, en tanto indica que prácticamente la mitad de los entrevistados no ha recibido información alguna respecto a su protección de salud ocupacional en su actual empleo. Esto de acuerdo a estudios en Chile es señalado como una barrera en el acceso a prestaciones de salud fruto de enfermedades profesionales o accidentes laborales ${ }^{12}$.

El aspecto más crítico radica en el escaso dominio de los beneficios que conlleva el ser parte de una mutual. Es decir, se sabe en qué consiste una mutual, pero no la especificidad de los derechos que conlleva ser parte de una. Esto adquiere una particular urgencia, ya que los datos a nivel internacional, dan cuenta de que los trabajadores, aun cuando consideran importante la seguridad, no toman acciones concretas para mejorarla ${ }^{17}$, lo que puede ser explicado por la percepción de que las empresas se encuentran preocupadas seriamente de este tema, restando así importancia a la iniciativa individual. Esto hace más relevante aún el aumentar los esfuerzos por parte de las mutuales para promover de forma más activa sus beneficios.

Es relevante también mencionar las diferencias en la valoración respecto a la información recibida por parte de las mutuales. Mientras la información de beneficios no relacionados con la salud ocupacional es altamente valorada (5.4 de 7 puntos), la información respecto a prevención de riesgos ocupacionales posee una baja valoración (4.1 de 7). Esta baja valoración de la información respecto a la prevención de riesgos (una de las razones de ser de las mutuales) abre una línea de investigación respecto a cómo hacer más amena y entretenida la provisión de información sobre prevención de riesgos laborales a los trabajadores. La baja valoración de este elemento crítico para la reducción de accidentes cons- tituye un llamado de atención respecto a cómo transmitir de forma eficiente comportamientos que faciliten el trabajo seguro.

Por otro lado, entre los aspectos más relevantes entregados por esta mirada exploratoria está el hecho de que el 19\% de los trabajadores entrevistados señale haber sido víctima de un accidente laboral o enfermedad profesional. Esta cifra da cuenta de una visión distinta a la entregada por las estadísticas oficiales, que para el año 2012 reportó tasas cercanas al 6\% en el caso del primer tipo de incidente, y el 11\% para el segundo.

Por otro lado, resulta preocupante que el 18\% de los entrevistados que reportan haber sufrido algún tipo de accidente señalen que su atención fue "complicada, debido a que hubo que comprobar ante la mutual que fue un accidente laboral". Esto debiera ser objeto de estudios de seguimiento de trabajadores usuarios del seguro de accidentes laborales con el fin de identificar la existencia de trabas en el ejercicio de este derecho y avanzar en su remoción.

En el caso de aquellos trabajadores que recibieron atención por concepto de enfermedad profesional, se aprecia una satisfacción mayor con aspectos relacionados a la atención médica recibida y a los planes de rehabilitación que con los aspectos administrativos $\mathrm{y}$, en particular, con los procedimientos de traslado hacia los centros asistenciales.

Con el objeto de realizar un adecuado balance de los resultados expuestos es necesario dar cuenta de las limitaciones de la aproximación empírica de este estudio. La primera está asociada con la recolección de la información, ya que existen dificultades en la realización de encuestas vía telefónica, no sólo por posibles sesgos relativos a la posesión de este medio de comunicación, sino también por la alta inestabilidad en la propia aplicación. En la medida que no pueden utilizarse elementos para apoyo visual, las categorías de respuesta deben mantenerse en un número que permita al encuestado recordarlas, lo que no permite realizar aproximaciones más complejas. Y, en segundo lugar, la muestra está acotada a hogares del Gran Santiago, lo que puede ser un factor que permita comprender algunas de las diferencias halladas con estudios realizados a nivel nacional.

A pesar de estas dificultades, los resultados muestran la necesidad de ahondar en la difusión de los derechos de los trabajadores en materia de seguridad y salud laboral, por la especial relevancia que tiene, desde el punto de vista del bienestar de los individuos, así como del crecimiento económico del país.

Finalmente, es necesario destacar la necesidad de realizar mayores investigaciones sobre el sistema de salud laboral, tanto público como privado, más allá de esta aproximación exploratoria. Esto permitirá identificar puntos críticos para la difusión de los derechos de los afiliados a las mutuales de seguridad, así como posibles factores explicativos del conocimiento o desconocimiento de estos. En esta línea, el desarrollo de estudios orientados a evaluar la efectividad de metodologías de capacitación en prevención de riesgos laborales permitirá identificar prácticas más eficientes de capacitación en esta importante área. De igual forma, permitirá conocer qué aspectos requieren ser revisados o mejorados para facilitar el uso del seguro por parte de los usuarios del sistema.

\section{Fuente de financiamiento de la investigación:}

Este estudio fue financiado por el Instituto de Salud Pública de la Universidad Andrés Bello. 


\section{Anexos}

ENCUESTA MUTUALES UNAB

Buenas tardes, mi nombre es.... y soy encuestador/a del Centro de Estudios Cuantitativos de la Universidad Andrés Bello. En este momento estamos realizando un estudio sobre seguridad laboral. Si usted es mayor de 18 años, ¿podría hacerle algunas preguntas? Esta entrevista tiene una duración aproximada de 6 minutos. La información entregada será completamente confidencial.

P1. ¿Actualmente usted se encuentra trabajando? (Marque sólo UNA alternativa)

$\mathrm{Si}$

No **

** SI P1 = 2 (NO TRABAJANDO), AGRADECER Y TERMINAR ENTREVISTA

P2. ¿Cuál de las siguientes situaciones caracteriza mejor su situación laboral? (Marque sólo UNA alternativa)

Con contrato indefinido

Con contrato a plazo fijo

Presta servicios a honorarios

Trabajador por cuenta propia

Temporero/a

P3. ¿Cuántas personas trabajan en esta empresa? (Anote número)

P4. ¿Cuál actividad desarrolla su empresa? (Marque sólo UNA alternativa)

\section{personas}

Producción de materias primas (mineria, agricolas)

Construcción

Comercio (retail)

Servicios (financieros, educación, salud, etc.)

Industria (producción de bienes/cosas)

Otra

P5. ¿Sabe en qué consisten las mutuales de seguridad? (Marque sólo UNA alternativa)

P6. ¿Sabe si su empresa está afiliada a alguna mutual de seguridad? (Marque sólo UNA alternativa)

No

No

Si, Instituto de Seguridad del Trabajo (IST)

Sí, Asociación Chilena de Seguridad (ACHS)

Si, Mutual de Seguridad (Cámara de Ch. la Construcción)

Sí, Instituto de Seguridad Laboral (ISL)

P7. ¿Conoce cuáles son los beneficios y cobertura de las mutuales de seguridad? (Marque sólo UNA alternativa)

P8. ¿Alguna vez ha utilizado alguno de los servicios proporcionados por su mutual?, ¿cuáles? (Marque TODAS las alternativas que correspondan)

No (pasar a P.16)

$S$ Si, descuentos (educación, tiendas)

Si, capacitación en prevención de riesgos

Sí, atención médica por enfermedades profesionales

Sí, atención médica por enfermedades comunes

Sí, infraestructura de rescate

Si, otra

P9. ¿Cómo supo que tenía derecho a ser atendido/a por la mutual? (Marque sólo UNA alternativa)

Capacitaciones realizadas por la mutual

Representante de la mutual en la empresa

Prevencionista de riesgos

Compañeros/as de trabajo

Medios de Comunicación

Otro

P10. De 1 a 7, ¿qué nota le pone usted a la información que la mutual le entrega para el uso de: (Si "no sabe", marcar 9)

1) Programa de beneficios

2) Cobertura de salud por enfermedad laboral

3) Cobertura de salud por enfermedad común

4) Beneficios familiares

5) Capacitación en prevención de riesgos

P11. Durante los últimos 12 meses, ¿usted ha sufrido alguna enfermedad y/o accidente laboral? (Marque sólo UNA alternativa)

P12. Con respecto a la prestación de servicios entregada, ¿cómo describiría el proceso? (Marque sólo UNA alternativa)

No (PASAR A P.16)

Complicada, ningún compañero/a de trabajo sabía qué hacer.

Complicada, hubo que comprobar ante la mutual que fue un accidente laboral.

Sencilla, existe un protocolo claro en la empresa para el traslado

de accidentados a la mutual

Sencilla, existe un protocolo claro en la empresa para el traslado

de accidentados a la clínica/consultorio

Otro

1) Tiempo de espera para traslado

P13. De 1 a 7, ¿qué nota le pone usted a los siguientes elementos de dicha atención? (Si "no sabe", marcar 9)

* En el caso de que el traslado haya sido de forma particular (auto de la empresa, etc.) se debe marcar 0

2) Calidad del traslado*

3) Procedimientos administrativos para atención

4) Recepción en el centro de salud (trato)

5) Calidad de la atención médica recibida

6) Programa de rehabilitación

P14. De 1 a 7, y según su experiencia, ¿cómo evaluaria usted la atención recibida?

P15. Según su experiencia, ¿usted considera que al momento de regresar a su trabajo estaba...? (Marque sólo UNA alternativa)

P16. Dado el caso que sufriese algún accidente laboral, ¿sabria qué hacer? (Marque sólo UNA alternativa)

Apto para volver a trabajar

No apto para volver a trabajar

No

Si, debo ir a la posta u hospital más cercano.

$\mathrm{Si}$, debo exigir seguro por accidente laboral en la mutual afiliada a la empresa.

No, no conozco la normativa

No, no hay normativa

Sí, debo atenderme particularmente (FONASA/ISAPRE)

$\mathrm{Si}$, debo recurrir a la mutual afiliada a la empresa

P17. ¿Conoce la normativa de su empresa respecto a los accidentes laborales? (Marque sólo UNA alternativa) 
Anexos (continuación)

\section{ENCUESTA MUTUALES UNAB}

P18. Si tuviera algún problema con la mutual, ¿a quién recurriría en busca de ayuda? (Marque sólo UNA alternativa)
A nadie
A la misma mutual
A su empresa
A su ISAPRE /FONASA
Al Ministerio de Salud
A la Superintendencia de Salud
A la Superintendencia de Seguridad Social
AI SERNAC

**Finalmente, y sólo para uso estadístico, me gustaría hacerle las siguientes preguntas:

P19. Sexo del encuestado (Marque sólo UNA alternativa. Completar automáticamente)

Hombre

Mujer

P20. Edad (al día de la encuesta) (Anote número)

P21. Nivel educacional (al día de la encuesta) (Marque sólo UNA alternativa)

Educación básica

Educación media

Educación superior: CFT / IP

Educación superior: Universidad Postgrado

*Sólo para el encuestador/a:

P22. Nombre del encuestador

P23. Fecha (DD/MM/AAAA)

P24. GSE (Ver Tabla)

C1
C2
C3

P25. Fono encuestado

P36. Región (Marque sólo UNA alternativa)

Región Metropolitana

$\checkmark$ Región

VIII Región

\section{REFERENCIAS}

1) Urriola, R. Chile: protección social de la salud. Rev Panam Salud Pública. 2006; 20; 273-286.

2) Robles, C. Sistemas de protección social en América Latina y el Caribe: Chile. CEPAL. Santiago. Chile. 2013. Recuperado en http://www.cepal.org/publicaciones/xml/9/48989/SPS_Chile_esp.pdf

3) Montoya, C. SSAP Seguridad Social Administrada por Privados. Visión general del complejo de la seguridad social administrada por privados. Chile 20002004. Cuadernos Medico-Sociales. 2004; 44: 81-90.

4) Goic, A. Armas, R. Descentralización en salud y educación: La experiencia chilena. Rev. med. Chile. 2003; 131: 788-798.

5) Miranda, E. Descentralización y privatización del sistema de salud chileno. Revista de Estudios Públicos. 1990; 39: 5-66.

6) Becerril-Montekio, V. Reyes, J.D. y Manuel, A. Sistema de salud de Chile. Salud Pública Mexico. 2011; 53: 132-143.

7) Hämäläinen $P$, Takala J \& Saarela KL. Global estimates of occupational accidents. Safety Science. 2006; 44: 137-156.

8) Hämäläinen, P. Saarela, K.L. y Takala, J. Global trend according to estimated number of occupational accidents and fatal work-related diseases at region and country level. Journal of Safety Research. 2009; 40: 125-139.

9) Giuffrida, A. lunes, R.F. y Savedoff, W. D. Occupational risks in Latin America and the Caribbean: economic and health dimensions. Health Policy and Planning. Oxford University Press. 2002: 17: 235-246.

10) Brahm, F. Singer, M. Valenzuela, L. Ramírez, C. Comparación internacional de sistemas de salud y seguridad laboral. PUC-0IT. Santiago. 2010.

11) Superintendencia de Seguridad Social. 2013. Estadisticas de Seguridad Social. Gobierno de Chile. Santiago. Chile. 2012.

12) Ministerio de Salud, Dirección del Trabajo e Instituto de Seguridad Laboral. Primera Encuesta Nacional de Empleo, Trabajo, Salud y Calidad de Vida de los Trabajadores y Trabajadoras en Chile. Gobierno de Chile. Santiago. Chile. 2011.

13) Rodríguez, J. Tokman, M. Licencias médicas: ¿Chilenos enfermizos? En foco Corporacion Expansiva. 2003; 16: 1-14. Recuperado en http://www.expansiva. cl/media/en_foco/documentos/01122003130631.pdf

14) Espinoza, V. Barozet, E. y Méndez, M.L. Estratificación y movilidad social bajo un modelo neoliberal: El caso de Chile. Revista Laboratorio. 2011; 25. Recuperado en http://www.desigualdades.cl/wp-content/uploads/2010/11/Espinoza-BarozetMendez-Estratificaci\%C3\%B3n-Lavboratorio.pdf

15) Vives, A. Jaramillo, H. Salud Laboral en Chile. Arch Prev Riesgos Labor. 2010; 13: 150-156.

16) Elgood, J. Gilby, N. y Pearson, H. Attitudes toward health and safety: a quantitative survey of stakeholder opinion. MORI Social Research. Institute report for the Health Safe Executive. United Kingdom. 2004.

17) Braithwaite, V. Motivations, attitudes, perceptions, and skills: pathways to safe work. Safe Work Australia. 2011. Recuperado en http://www.safeworkaustralia. gov.au/sites/SWA/about/Publications/Documents/581/Motivation_Attitude_ Perceptions_and_Skills_Pathways_to_Safe_Work.pdf 